9-1-2018

\title{
Engagement des hommes dans la planification familiale au Togo: évaluation des modèles de counseling de couples
}

Breakthrough RESEARCH

Follow this and additional works at: https://knowledgecommons.popcouncil.org/departments_sbsr-rh How does access to this work benefit you? Let us know!

\section{Recommended Citation}

Breakthrough RESEARCH. 2018. "Engagement des hommes dans la planification familiale au Togo: évaluation des modèles de counseling de couples," Activity brief. Washington, DC: Population Council. 


\section{Engagement des Hommes dans la Planification Familiale au Togo}

\section{Évaluation des Modèles de Counseling de Couples}

Breakthrough RESEARCH évalue les services de counseling fournis aux couples en groupes et à domicile dans le district de Kloto au Togo afin de déterminer les avantages de chaque approche et l'aptitude à une mise à l'échelle de ces approches. Dans ce district, le projet Survie de la Mère et de l'Enfant de I'USAID a conçu des programmes portant sur l'engagement des hommes et le counseling des couples. Ces programmes doivent être évalués avant la mise en place d'un cadre commun de counseling.

\section{POURQUOI MENONS-NOUS CETTE ACTIVITÉ ?}

Vingt années de littérature ont montré que les normes liées au genre - ou les attentes sociales concernant les rôles et comportements des hommes et des femmes - affectent les décisions de planification familiale des couples. Par conséquent, un counseling efficace en planification familiale devrait engager les hommes et les femmes dans des discussions sur l'équité entre les sexes lors des prises de décision. Les programmes de counseling pour les couples ont eu des résultats particulièrement prometteurs à cette fin, améliorant globalement la communication entre les partenaires et leur utilisation de la planification familiale.

Dans le District de Kloto au Togo, le Projet Survie de la Mère et de l'Enfant a conçu plusieurs programmes pour l'engagement des hommes et le counseling des couples offrant un potentiel de mise à l'échelle. Les modèles doivent être évalués avant la mise en place d'un cadre commun de counseling dans la Région Ouest Africaine.

\section{COMMENT CETTE ACTIVITÉ SERA-T-ELLE MISE EN CEUVRE ?}

Breakthrough RESEARCH évalue deux types de counseling des couples afin d'estimer leurs avantages et leur faisabilité et déterminer chez chacun d'eux les éléments les mieux adaptés à un déploiement à une échelle plus large. Les deux modèles de counseling - de groupe et à domicile - seront mis en œuvre dans des cantons distincts du district de Kloto au Togo.

- Le canton de Kpadapé (4 villages) abritera le counseling de groupe. Des agents de santé communautaires (ASCs)

\section{APERÇU DES ACTIVITÉS}

Évaluer les coûts et avantages relatifs des deux méthodes de counseling de couples

Partenaire Principal

ESPACE GÉOGRAPHIQUE

Institute for Reproductive

Health (IRH) à I'Université de

Georgetown

Togo

CONTACT

Rebecka Lundgren à

CALendrier PRÉVu

$2018-2019$

animeront les sessions, combinant vidéos numériques et réflexion de groupe. Le counseling sera offert aux groupes mixtes et aux groupes de même sexe.

- Le canton de Kpimé (15 villages) accueillera les visites de counseling de couples à domicile. Des ASCs animeront les sessions en utilisant des cartes-conseils.

Sur les deux sites, les ASCs, les prestataires de service de santé et les conseillers seront formés sur quatre modules : planification familiale, communication au sein du couple; normes liées au genre; et violence par le partenaire intime.

L'équipe chargée de l'étude examinera trois questions sur chaque site:

- Quels processus et éléments sont nécessaires - y compris les ressources, les outils et les profils comportementaux des prestataires - pour déployer et évaluer avec succès les deux modalités de counseling de couples au Togo ? 
- Comment les couples prennent-ils leurs décisions et communiquent-ils différemment lorsqu'ils participent à un counseling individuel par rapport à un counseling de groupe (par exemple : plus de temps pour un dialogue lors du counseling individuel que lors d'un counseling de groupe) ?

- Dans quelle mesure le programme a-t-il amélioré la capacité perçue des couples à exprimer leur désir de planification familiale et d'utilisation négociée des contraceptifs ? Et dans quelle mesure les ASCs sont-ils capables de conseiller les couples sur la planification familiale?

Les méthodes suivantes seront utilisées pour répondre à ces questions :

- Des interviews approfondies avec 12 couples, segmentées par approche de counseling et caractéristiques démographiques (par exemple : âge, éducation, etc.), afin de comprendre les motivations et les expériences des couples concernant les sessions de counseling. Les interviews exploreront les désavantages, les effets potentiels sur l'équité entre les sexes lors des prises de décision, la capacité des femmes à opérer des choix en matière de reproduction, ainsi que d'autres résultats comportementaux, tels que l'utilisation de la contraception moderne et la résolution des conflits.

- Des interviews approfondies avec cinq ASCs et trois prestataires de service basés dans des formations sanitaires qui ont participé à la formation à l'intervention, afin de comprendre leurs expériences en matière de prestation de counseling. En utilisant des approches participatives, les interviews exploreront la manière dont les ASCs sont orientés et formés et comment les prestataires de service des formations sanitaires sont inclus dans l'intervention.

- Échanges entre les groupes de discussion (jusqu'à deux) et les chargés de mise en œuvre pour évaluer la faisabilité et la mise à l'échelle des approches de counseling.

\section{- Consultations avec les chargés de mise en œuvre, les par-} ties prenantes et le Comité d'Utilisation de la Recherche au Togo, en groupe ou individuellement. L'équipe chargée de l'étude documentera pour les deux approches de counseling un cadre conceptuel et la théorie de changement subséquente qui détermine la logique d'intervention et les voies à suivre en vue d'obtenir des résultats.

\section{PRODUITS ANTICIPÉS}

Rapport sur les études de cas

Guide pour l'élaboration du suivi et évaluation des outils évaluant les approches de counseling

Échelles d'auto-efficacité pour l'évaluation de la communication au sein des couples et de la capacité des prestataires à conseiller les couples

\section{COMMENT LES RÉSULTATS CONTRIBUERONT-ILS À L'AMÉLIORATION DES PROGRAMMES CSC ?}

La littérature examinée à ce jour révèle que le counseling des couples peut améliorer une gamme de résultats de santé sexuelle et reproductive (SSR), y compris l'utilisation de la planification familiale, la communication au sein du couple et le soutien au partenaire en ce qui concerne l'utilisation de la planification familiale, la connaissance de la fécondité et de la planification familiale, ainsi que la participation des hommes aux services de counseling et de SSR.

Le Projet Survie de la Mère et de l'Enfant de I'USAID a conduit avec succès des programmes de counseling en planification familiale individuels et de groupe au Togo. Cependant, les avantages et les inconvénients de ces modèles doivent être évalués avant de poursuivre la mise à l'échelle. Le Togo a un taux élevé de fécondité (4,8 enfants par femme), un faible taux utilisation des contraceptifs (20\% des femmes mariées âgées de 15 à 49 ans) et un taux élevé de besoins non satisfaits en planification familiale (34\%). Pour améliorer ces statistiques de façon significative, les programmes de santé reproductive doivent identifier un cadre de counseling mieux adapté à ce contexte.

\section{Breakthrough RESEARCH}

Cette activité est mise en œuvre dans le cadre de Breakthrough RESEARCH. Breakthrough RESEARCH suscite un changement social et comportemental (CSC) en menant une recherche et une évaluation de pointe et en promouvant des solutions basées sur des données probantes en vue d'améliorer les programmes de santé et de développement à travers le monde. Breakthrough RESEARCH est un consortium dirigé par Population Council en partenariat avec Avenir Health, ideas42, Institute for Reproductive Health à I'Université de Georgetown, Population Reference Bureau, et I'Université de Tulane.

\section{Remerciements}

Breakthrough RESEARCH a été rendu possible grâce au généreux soutien du people Américain à travers l'Agence des États-Unis pour le Développement International (USAID) selon les termes de l'accord de coopération no. AID- OAA-A-17-00018. Le contenu de ce document relève de l'unique responsabilité de Breakthrough RESEARCH et Population Council et ne reflète pas nécessairement l'opinion de I'USAID ou du Gouvernement des États-Unis.

\section{Breakthrough RESEARCH}

Population Council

4301 Connecticut Avenue, NW

Suite 280

Washington, DC 20008

Tel: +1 2022379400

breakthroughactionandresearch.org

Références pour les Photos

(c) 2016 Sarah Hoibak/VectorWorks, Avec l'aimable autorisation de Photoshare 